\title{
Complex method for angular-spectral analysis of volume phase diffraction gratings recorded in photopolymers
}

\author{
P. Vojtís̃ek \\ vojtisek@ipp.cas.cz
}

M. Květoň

kveton@holoplus.cz

I. Richter

ivan.richter@fjfi.cvut.cz

\author{
Institute of Plasma Physics AS CR, v.v.i., Centre TOPTEC, Sobotecká 1660, 511-01, Turnov, Czech Re- \\ public \\ Czech Technical University in Prague, FNSPE, Department of Physical Electronics, Břehová 7, 115-19, \\ Prague 1, Czech Republic \\ Holoplus s.r.o., Nademlejnské 1, 19800 Prague 9, Czech Republic
}

Czech Technical University in Prague, FNSPE, Department of Physical Electronics, Břehová 7, 115-19, Prague 1, Czech Republic

Photopolymer recording materials are nowadays widely used for recording of diffraction gratings and other diffraction elements. For obtaining the best performance of these diffraction gratings for desired applications, it is important to assess these gratings from many different perspectives. In this contribution, we present an experimental and characterization approach to an analysis of diffraction gratings recorded into photopolymer materials. This approach is able to provide a complex and very illustrative description of these gratings response and, with accordance to the theory, information about some important grating parameters, such as a spatial period, slant angle, etc., as well. This approach is based on the measurement of a grating response for a wide range of angles and wavelengths and then on the construction and subsequent analysis of maps in the angular-spectral plane. It is shown that the measurements are in a good agreement with the theoretical predictions based on either approximate (Kogelnik's coupled wave theory) or rigorous (RCWA) techniques and that this approach provides complex and detailed characterization of the grating response which can be used for additional optimization or decision of applicability of measured sample gratings.

[DOI: http://dx.doi.org/10.2971/jeos.2016.16009]

Keywords: Photopolymers, diffraction gratings, angular-spectral maps, spectral selectivity, angular selectivity

\section{INTRODUCTION}

For recording of information coded into an interference pattern, wide range of different materials can be used. One class of these recording materials are photopolymer based materials where the storing of information is done by a light induced polymerization of monomers [1]. Recently, these recording materials based on polymerization of primary units (monomers) were studied to a great extend - theoretically (description of the grating formation [2]-[4], approach to the description of wide range of properties [5]-[7], etc.) and experimentally (material composition [8]-[11], measurement of parameters [12]-[15], etc.).

In our laboratory, we have prepared and tested this type of a recording material through the response to exposure of a harmonic interference field [16]. Photopolymer is a selfdeveloping recording medium and; hence, a volume phase grating is formed already during the holographic exposure. Current experimental and commercial photopolymers such as Bayfol HX [10] are very effective recording media and effects of grating shrinkage, overmodulation or formation of higher diffraction orders may correspondingly occur. For the analysis of these effects in volume phase gratings, we have developed a complex method for grating parameters characterization and extraction. As we suppose that the volume grating has only limited number of orders (ideally, only the first and zeroth order) and the grating is illuminated with white light, we can obtain from the measurement of the transmitted light (the zeroth order) for different incident angles a map characterizing angular and spectral behavior of the thick grating.

The aim of this paper is, firstly, to present and discuss an approach based on angular-spectral measurements for the characterization of diffraction volume phase gratings and also, secondly, to show that the results of measurements are in a good agreement with the theory. Based on this agreement between the measurement and theory, it will be illustrated that it is possible to obtain various characteristic grating and experimental parameters. Further, the paper aims to show that this approach provides complex and illustrative description of the grating from many different perspectives. Usually, for the analysis of diffraction gratings, either the angular [14, 17, 18] or wavelength/spectral [19] selectivity (scan) is considered and measured. Diffraction effects and grating parameters are then obtained from these measurements. But this approach, which includes only one of these perspectives (angular or wavelength), leads to the loss of the more complex description and illustrative presentation of results. If both the angular and spectral selectivity are considered as in our case further, an 
angular-spectral map (AS map) can be obtained (either from simulations or from measurements). Moreover, wide range of information can be gained only by a quick overview of the AS map.

For the theoretical description of the diffraction behavior of the gratings, which would act as a comparison to the experimental data, Kogelnik's coupled wave theory [20] can be used. If it is used in its basic form, only the zeroth and the first diffraction orders can be analyzed and the AS map does not describes effects of higher diffraction and refractive index modulation orders. For obtaining information about the higher orders, the extended Kogelnik's coupled wave theory $[21,22]$ or a more rigorous approach such as a Rigorous Coupled Wave Analysis (RCWA) [23, 24], which calculates the AS maps numerically, can be used. The advantage of the Kogelnik's approach is a better physical insight into the problem, but the cost is less rigorous description of the diffraction problem and loss of some effects (e.g. higher diffraction orders, the influence of a more complex grating structure, etc.).

For the measurement, we have built a setup composed of collimated white-light source and motorized rotational stage with a sample of a grating. The transmitted light is measured with fiber spectrometer which is computer controlled just as the rotational stage. After the processing of collected data, the same AS map as in the theoretical case is obtained and can be compared and analyzed.

\section{THEORETICAL BACKGROUND}

The diffraction inside volume phase diffraction gratings is a consequence of the periodical spatial refractive index modulation. The spatially harmonically variable value of the refractive index can be described by a relation

$$
n(\mathbf{r})=n_{0}+n_{1} \cos (\mathbf{K} \cdot \mathbf{r})-n_{2} \cos (2 \mathbf{K} \cdot \mathbf{r})+\ldots,
$$

where $n_{0}, n_{1}, \mathbf{K}$ and $\mathbf{r}$ are the average refractive index, amplitude of the first refractive index modulation order, wave vector of the grating, and vector of position, respectively. The values of $n_{i}$ for $i>1$ represent amplitudes of higher refractive index modulation orders.

An optimal behavior (the highest diffraction efficiency) of these volume diffraction gratings is usually described by the Bragg condition (synchronization condition), which relates grating (characterized by a grating vector $\mathbf{K}$ ) and reconstruction parameters for the best energy transfer from a reconstruction (wave vector $\mathbf{k}_{\mathbf{r}}$ ) into a diffracted (wave vector $\mathbf{k}_{\mathbf{s}}$ ) wave. This condition can be written as $\mathbf{k}_{\mathbf{s}}=\mathbf{k}_{\mathbf{r}}-\mathbf{K}$ (Figure 1). If the Bragg condition is not fulfilled, then the energy transfer is not in its optimum and, consequently, the diffraction efficiency of the grating will change. For a wide range of wavelengths, the Bragg condition provides a corresponding angle which leads to a curve (Bragg curve) in the angular-spectral plane. If a plane wave reconstruction and a non-slanted transmission grating is assumed, then the Bragg condition, which for a given spatial period $\Lambda$ and a recording material (characterized by an average refractive index $n_{0}$ ) relates the Bragg angle $\Theta_{0}$ (inside the material) and the Bragg wavelength $\lambda_{0}$,

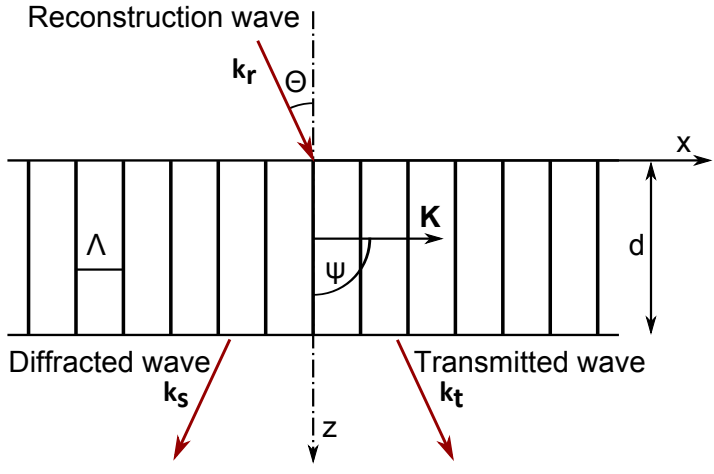

FIG. 1 Schematic illustration of a non-slanted transmission grating and its main characteristic properties (spatial period $\Lambda$, grating vector $\mathbf{K}$, thickness $d$, slant angle $\psi$, reconstruction angle $\Theta$, and position of reconstruction/incident, diffracted and transmitted waves). $\mathbf{k}_{\mathbf{r}}, \mathbf{k}_{\mathbf{s}}$, and $\mathbf{k}_{\mathbf{t}}$ denote the wave vectors of reconstruction/incident, signal/diffracted, and transmitted waves, respectively.

can be simplified to a relation $\Theta_{0}= \pm \arcsin \left[\lambda_{0} /\left(2 n_{0} \Lambda\right)\right]$ (Figure 2(a)).

If the mentioned angular-spectral plane of the grating response is filled for all angles and wavelengths with values of transmittance of the grating ( $\mathrm{z}$ axis), then the AS map is obtained (Figure 2(b)). This AS map then describes a transmittance response of the grating for a wide variety of different reconstruction parameters (angles, wavelengths, and possibly even polarizations). In more detail, Figure 2(a) represents a graphic interpretation of the phase synchronization condition in the angular-spectral plane for a case of a transmission grating (Figure 1) with a $700 \mathrm{~nm}$ spatial period and 90 deg slant angle. For this type of a grating two symmetrical curves with an axis of the symmetry at $0 \mathrm{deg}$ are obtained. At each point of this curve, the Bragg condition is fulfilled and the energy transfer between the incident and diffracted waves (or vice versa) is the most effective. If the reconstruction angles and wavelengths are not positioned at these Bragg curves, then the phase synchronization is not optimal and the energy transfer into the diffracted wave changes. This energy transformation for all possible reconstruction angles and wavelengths is shown in Figure 2(b) and it represents above mentioned AS map. The main valley in the AS map coincides with the Bragg condition (curves in Figure 2(a)). Furthermore, from the AS map it is possible to extract the transmittance for a wide range of reconstruction parameters, optimal reconstruction parameters (angle and wavelength where the transmittance is zero), and additional effects (e.g. higher diffraction orders, overmodulation), as will be shown below. Generally, this is only one type of possible response maps [25], but it is sufficient for a description of many grating properties.

For the description of diffraction properties of diffraction gratings, approximate theories, such as the Kogelnik's coupled wave theory [20], can be used under some conditions which are listed, for example, in original Kogelnik's paper [20].

For a relatively wide range of gratings, the condition of a small deviation from the Bragg condition can be little loosened because the Kogelnik's theory can describe the qualitative behavior with some error, even for larger deviation from the 


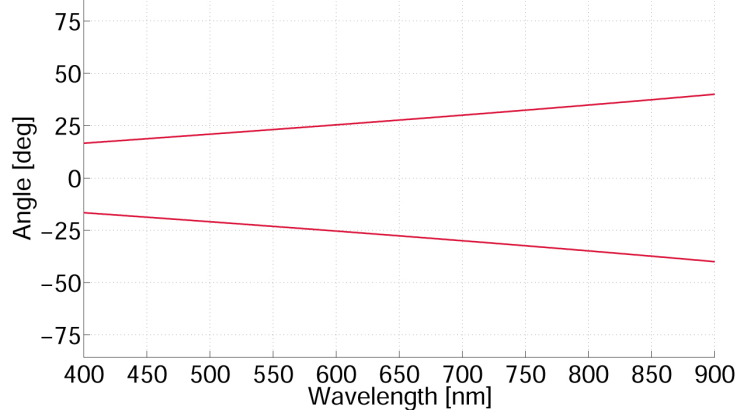

a)

b)

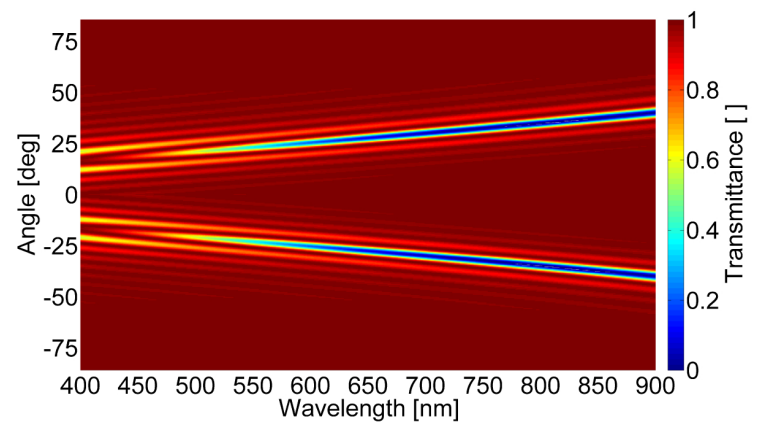

FIG. 2 Illustration of a) graphic interpretation of the Bragg condition in an angularspectral plane (Bragg curve), and b) the theoretical prediction (Kogelnik's theory) of the AS map of a non-slanted grating ( $\psi=90 \mathrm{deg})$ transmittance for $\Lambda=700 \mathrm{~nm}$, $n_{0}=1.52, n_{1}=0.025$, and thickness $\mathrm{d}=15 \mu \mathrm{m}$.

Bragg condition. For quantitative information and a more precise description of the qualitative behavior, a more rigorous approach has to be used, for example the Rigorous Coupled Wave Analysis (RCWA) [23, 24].

The Kogelnik's theory is here used mainly because it provides a more illustrative description and a better physical insight into the studied diffraction problem. Nevertheless, if the basic Kogelnik's theory is not extended to include higher diffraction and refractive index modulation orders, it gives information only about the zeroth and the first diffraction orders.

For the construction of the AS maps, the Kogelnik's theory describes the diffraction properties (efficiency $\eta$, transmittance $\tau$ ) of lossless dielectric transmission gratings via the relations

$$
\eta=\frac{\sin ^{2}\left(\phi \sqrt{1+\frac{\chi^{2}}{\phi^{2}}}\right)}{1+\frac{\chi^{2}}{\phi^{2}}}, \quad \tau=1-\eta
$$

where $\phi$ is defined as

$$
\phi=\frac{\kappa d}{\left(c_{r} c_{S}\right)^{\frac{1}{2}}},
$$

where $\kappa$ represents the coupling constant which is defined as $\kappa=\pi n_{1} / \lambda$ for the transverse-electric (TE) polarization. In these relations, $n_{1}$ stands for refractive index modulation (i.e. $\left.n=n_{0}+n_{1} \cos (\mathbf{K} \cdot \mathbf{r})\right), d$ stands for the thickness of the grating, $\lambda$ is the reconstruction wavelength (in free space), and $c_{r}=\cos (\Theta)$ and $c_{S}=\cos (\Theta)\left(\lambda / n_{0} \Lambda\right) \cos (\psi)$ represent the directional cosines of the reconstruction and the diffracted waves respectively $(\Theta$ is defined inside the medium). $\psi$ indicates an angle of a slant which is defined as the angle between the direction of the grating vector and the normal to the surface (Figure 1).

The parameter $\chi$ in Eq. (2) characterizes a detuning of the reconstruction from the Bragg condition

$$
\chi=\frac{\xi d}{2 c_{s}}
$$

where $\xi$ is defined as

$$
\xi=-K\left[\Delta \Theta \sin \left(\Theta_{0}-\psi\right)+\frac{\Delta \lambda}{2 n_{0} \Lambda}\right],
$$

where $K=(2 \pi) / \Lambda$ stands for the amplitude of the wave vector of a grating (i.e. $K=|\mathbf{K}|$ ), $\Delta \Theta=\left|\Theta_{0}-\Theta\right|$ is a difference of the Bragg angle $\Theta_{0}$ and the angle of the measurement/reconstruction $\Theta, \Delta \lambda=\left|\lambda_{0}-\lambda\right|$ indicates the detuning from the Bragg wavelength $\lambda_{0}$, and $\Lambda$ denotes the spatial period of the grating.

For reflection gratings, the definition of the relation for the diffraction efficiency has to be altered. The efficiency and transmittance are in the case of reflection gratings given as

$$
\eta=\left(1+\frac{1-\frac{\chi^{2}}{\phi^{2}}}{\sinh ^{2}\left(\phi \sqrt{1-\frac{\chi^{2}}{\phi^{2}}}\right.}\right)^{-1}, \quad \tau=1-\eta,
$$

where the parameters (real valued) $\phi$ and $\chi$ are redefined as follows

$$
\phi=\frac{i \kappa d}{\left(c_{r} c_{S}\right)^{\frac{1}{2}}}
$$

and

$$
\chi=-\frac{\xi d}{2 c_{s}}
$$

All used parameters have the same meaning as above. The reason for this redefinition, in the case of reflection gratings, is that the $c_{S}$ parameter is negative.

For a special case of gratings reconstructed under the Bragg condition, the diffraction efficiency is described as

$$
\eta=\sin ^{2}(\phi)
$$

for transmission gratings and as

$$
\eta=\tanh ^{2}(\phi)
$$

for reflection gratings.

Usually, when the gratings are studied from the perspective of their selectivity (detuning from the Bragg condition), only one cause is considered, i.e. either detuning via an angle or via a wavelength shift. In more general case, the deviation can be caused by both the angle and wavelength at the same time.

Because we are interested in the AS maps spanning near full angular scan, i.e. at least $\pm 80 \mathrm{deg}$, respectively $\pm 40 \mathrm{deg}$ when recalculated into the material with an average refractive index of $\sim 1.52$, it is necessary to take care of the definition of the angles and their orientation (especially, the slant angle). 


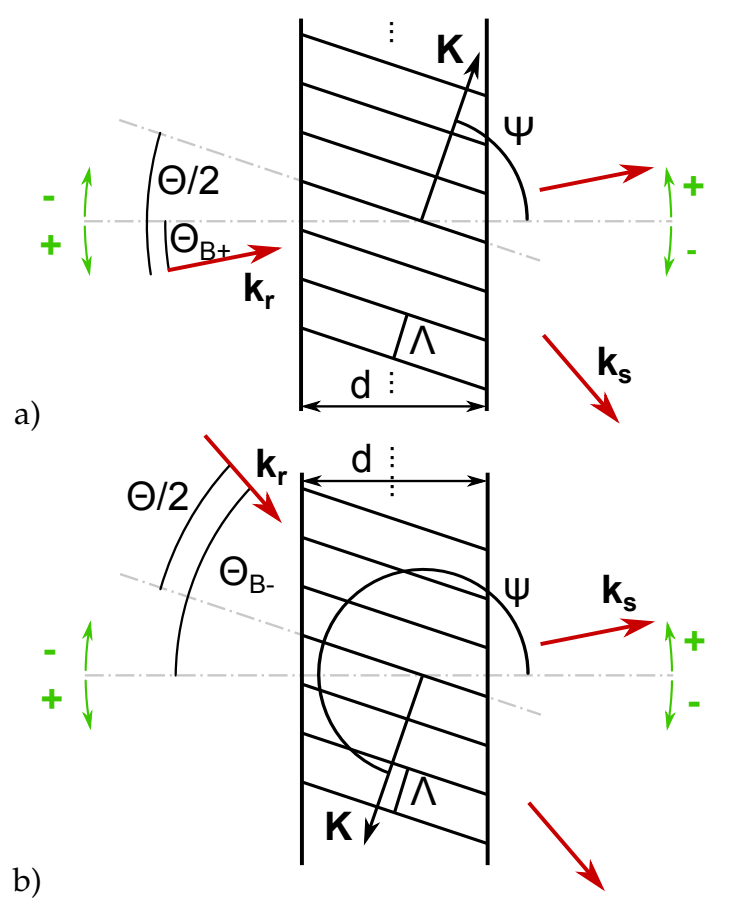

FIG. 3 Schematic illustration of the definition of angles in the case of the slanted grating for two cases of reconstruction. Reconstruction under a) "+" angle, and b) under "" angle. $\mathbf{k}_{\mathbf{r}}, \mathbf{k}_{\mathbf{s}}$ stand for wave vectors of the reconstruction and signal (diffracted) waves respectively. Green colored arrows indicate the sign of angles.

For the reconstruction carried out under the angles denoted as "+" (Figure 3(a)), the slant angle is $\psi$ and the Bragg angle $\Theta_{B+}=\Theta / 2-90+\psi$. For the angles denoted as "-" (Figure $3(b))$, the Bragg angle is $\Theta_{B-}=-(\Theta / 2+270-\psi)$.

The extension of the approximate approach to include higher (second) orders (diffraction and modulation) can be done following, for example, literature in Ref. [21] or [22]. Unfortunately, both these works address only the diffraction under the Bragg condition and do not include effects of detuning from this condition.

It is good to mention that the problem of the higher orders, both modulation and diffraction, is more pressing in the case of transmission gratings. In the case of reflection gratings, the spatial period is small and, therefore, there is smaller tendency to create higher modulation orders (a spatial resolution of the material is limited), which are usually the main contributors to the higher diffraction orders [21].

For a more rigorous description of the diffraction problems and for a generation of the AS maps, a numerical approach based on the RCWA method was used. In the case of the RCWA, the grating and the electromagnetic fields are represented as a sum of spatial harmonics [26]. The grating is spatially divided into elements (usually layers in the direction of propagation, but for volume gratings this division has to be done even in the direction perpendicular to the surface, so the spatial change of the refractive index is included); for two types of volume gratings (transmission and reflection), this division is illustrated in Figure 4. In each of these elements, the electromagnetic fields (modes) are expressed and propagated. At each interface, boundary conditions are applied.

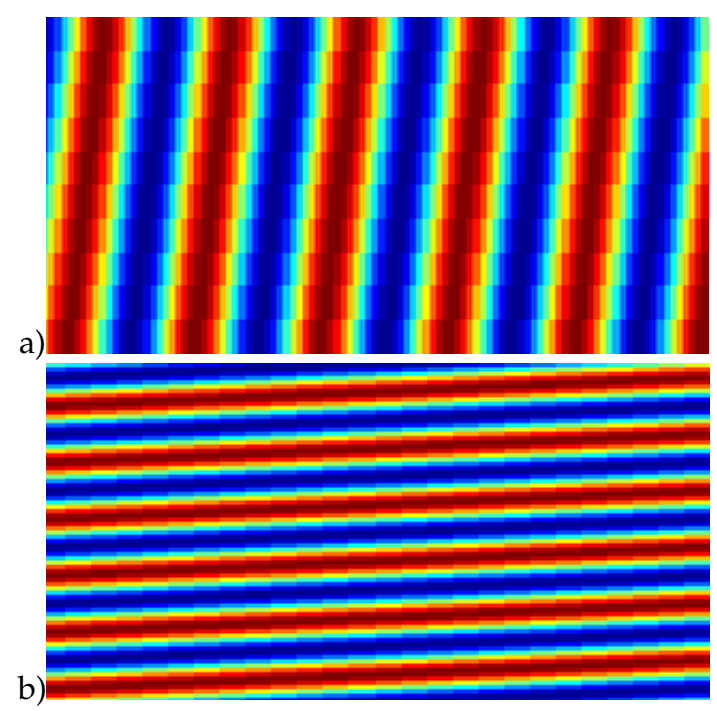

FIG. 4 Illustration of the division of the grating profile for the use in the RCWA. a) Transmission slanted grating, b) reflection slanted grating (an interface material-air is at the top and bottom of these figures). For calculations, used division is finer.

The drawbacks of the RCWA, compared to the Kogelnik's theory, are more complex and time consuming calculation and smaller direct physical insight. On the other hand, these are compensated by a possibility to get information about more effects; for example, higher diffraction orders and their behavior, presence of higher modulation orders $\left(n_{2}, n_{3}, \ldots\right)$, impact of the interface or multiple interfaces, more complex material composition and structure, and many others.

\section{RESULTS OF SIMULATIONS}

With the two approaches (Kogelnik's coupled wave theory and RCWA) mentioned in Section 2, simulations of the behavior of diffraction gratings were carried out. Based on these results, grating characteristic properties and effects (e.g. Bragg condition, spectral and angular selectivity, spatial period, slant angle, etc.) can be estimated. The resulting AS maps for sample transmission gratings (TE polarization (for TM polarization the concept of the AS maps can be used as well but it will not be here considered), angle scan \pm 85 deg, wavelength scan $400-900 \mathrm{~nm}$; these values were chosen so they would correspond to measurement limitations or will be little over these limitations) are shown in Figures 2(b), 5-6 and for reflection grating in Figure 7. In the case of the transmission gratings, following parameters were used: spatial period $\Lambda=700 \mathrm{~nm}$, average refractive index $n_{0}=1.52$, refractive index modulation $n_{1}=0.025$ (no higher orders of the modulation were considered, i.e. $n_{i}=0$, for $i>1$ ), thickness of the grating $d=15 \mu \mathrm{m}$, and two slant angles $\psi=90$ and $70 \mathrm{deg}$. For reflection gratings, parameters $\Lambda=175 \mathrm{~nm}, n_{0}=1.52$, $n_{1}=0.02,(\mathrm{~d}=15 \mu \mathrm{m})$, and $\psi=10 \mathrm{deg}$ were considered. Values of these parameters were chosen so they would correspond to typical sample gratings used in practice.

These calculations were done inside the material and then the interface of the material and air (as in a real measurement) was included by a re-computation (re-sampling) of the incidence angles through the use of the Snell's law. Fresnel angu- 


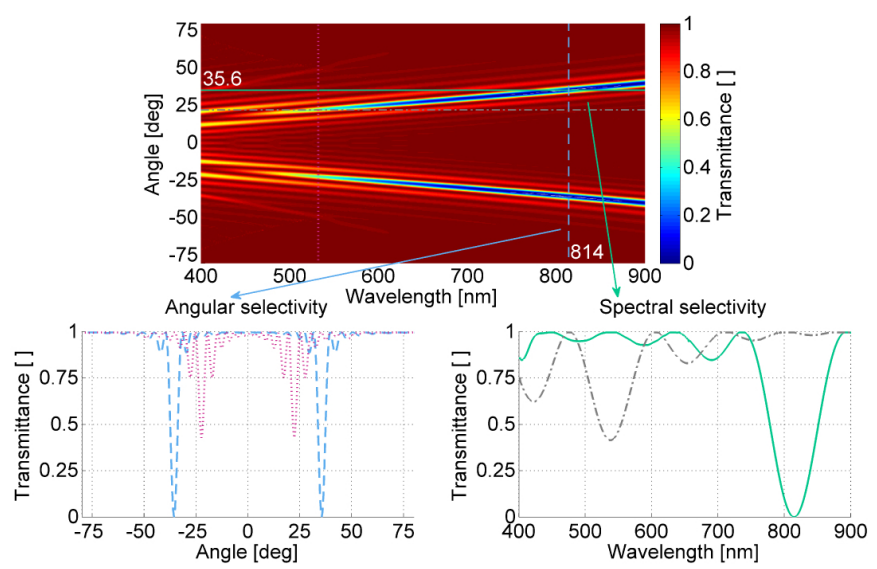

FIG. 5 AS map of a total transmittance of a diffraction grating obtained from the RCWA and an illustration of two angular and spectral sensitivities - curves with (blue/dashed and green/solid curve) and without (purple/doted and gray/dash-doted) optimal reconstruction parameters for the given transmission grating. $\Lambda=700 \mathrm{~nm}, n_{0}=1.52$, $n_{1}=0.025, d=15 \mu \mathrm{m}, \psi=90 \mathrm{deg}$.

a)
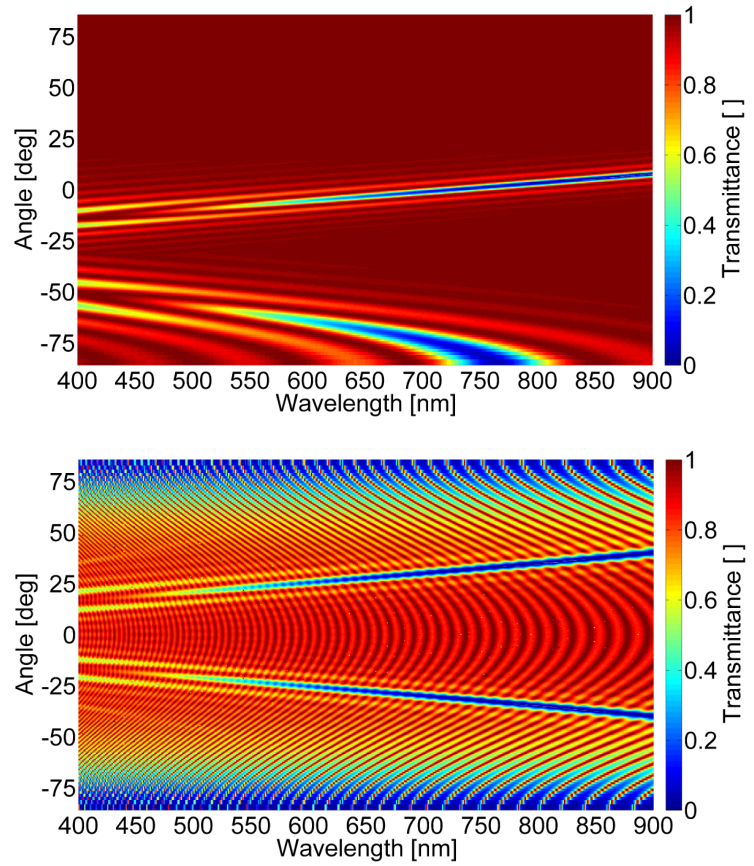

FIG. 6 a) Calculated AS map (Kogelnik's theory) for a transmission grating with following parameters: $\Lambda=700 \mathrm{~nm}, n_{0}=1.52, n_{1}=0.025, d=15 \mu \mathrm{m}, \psi=70$ deg. b) Illustration of material-air interfaces inclusion on the AS map for a slanted transmission grating. ( $\Lambda=700 \mathrm{~nm}, n_{0}=1.52, n_{1}=0.025, d=15 \mu \mathrm{m}, \psi=90 \mathrm{deg}$ ).

lar reflection losses were not included, because the measurement will be compensated for them. The effect of the interface (multiple interferences due to index boundaries) was included only in one case (Figure 6(b)) for a comparison with the aim to find out how or if the profile (e.g. position of the Bragg condition) of the AS maps changes.

The comparison between the results of the Kogelnik's theory (Figure 2(b)) and RCWA (Figure 5) indicates a good agreement in the description of the overall profile of the AS maps which includes the main trends such as the position of the Bragg condition, the spectral and angular profiles, the bending of the Bragg condition profile, etc. Nevertheless, there are two main disagreements, the first one is based on the presence of higher diffraction orders (for RCWA results even when no higher modulation orders were assumed), the second one is the distribution of side lobes (side peaks of the transmittance corresponding to the studied diffraction order) - for both approaches, the trend and character of these side lobes is approximately the same but the exact position differs.

Generally, the transmission gratings main characteristics indicated in the AS maps (Figure 5) can be summarized as follows.

The Bragg condition is fulfilled for a limited angular range and for the full range of wavelengths (the range of wavelengths is limited by the spatial period of the grating and the interface air-material). It is, from the angular perspective, fulfilled twice and is symmetrical for the non-slanted gratings with the axis of the symmetry positioned at $0 \mathrm{deg}$.

The slant of the grating shifts the profile to the side (from an angular perspective) and the interface material-air causes a bending of the profile (Figure 6(a)). For sufficiently large slant angles, an in/out-coupling behavior is present for longer wavelengths than is the wavelength fulfilling the Bragg condition for a total reflection. This is indicated by an "unpaired" Bragg condition in the AS maps. Spatial period of the grating influences the openness of the V-like shape, visible in the AS map.

Angular and spectral cross-sections provide information about the sensitivity (selectivity) to the angular (for a given wavelength) or wavelength (for a given angle) detuning from the Bragg condition; it is indicated in Figure 5 for two different wavelengths and angle cross-sections. From the FWHM of the cross-section curves follows that the selectivity to the angular detuning is higher than to the wavelength. The angular cross-section is more perpendicular to the curve representing the Bragg condition and, consequently, is narrower. Oppositely, the wavelength cross-section is more parallel to this curve and has broader profile.

Local decreases in the transmittance in the AS map may indicate a transformation of light into a diffraction order. The actual presence has to be confirmed based on additional information, e.g. an expected position of the diffraction order.

Optimal reconstruction parameters (the angles and wavelengths at which the grating pronounces maximal diffraction into the first diffraction order) are indicated as a region around the minimum in the AS map along the Bragg condition (e.g. the central wavelength and angle of this region $\lambda_{o p t}=814 \mathrm{~nm}$ and $\Theta_{\text {opt }}=35.6 \mathrm{deg}$ in Figure 5).

Transmittance profile along the Bragg condition is not constant. It is possible to find a zero transmittance in the AS map along the Bragg condition (optimal reconstruction) and the transmittance for a different Bragg condition than this optimal reconstruction is increasing on both sides from this optimal reconstruction. More precisely, as the wavelength gets shorter the transmittance gains a value of one and then drops to values near zero again (effect of overmodulation [17, 27]). 
Moreover, the AS maps indicate even overmodulation [27] of the gratings, or more precisely, the reconstruction parameters for which the grating will behave as overmodulated. This property is connected not only to the reconstruction parameters, but to the material and the recording parameters as well. Nevertheless, if the grating is recorded, it is possible to check whether the grating is overmodulated or not for the desired reconstruction parameters. This overmodulation is indicated by an increase of the transmittance in the main peak under the Bragg condition and by the decrease of the transmittance of the side lobes [17,27]. For the grating presented in Figure 5, the region of the overmodulation is for wavelengths shorter than $814 \mathrm{~nm}$ and corresponding angles.

Furthermore, two properties of the angular selectivity, which are not as readily apparent from only the angular or spectral scans, are illustrated. The first is that, as the wavelength is getting longer, the angular difference of the angles fulfilling the Bragg condition is getting bigger. It has a symmetrical V-like profile for non-slanted gratings. The second is connected to the side lobes of the profile. From the perspective of shorter wavelengths, the number and influence of the side lobes in the angular selectivity is increasing. This is the consequence of a dependency on the $\lambda$ in Eq. (3).

From the results calculated with the RCWA (Figure 5), the second diffraction order and its behavior can be observed as well; for example, its position, its angular shift or bending as the wavelength grows, its extinction for longer wavelengths, etc. If higher modulation orders (e.g. $n_{2}, n_{3}$ ) are included into the calculations, even higher diffraction orders (e.g. third) will be present. From information on these higher orders, it is possible to assess whether and to what extend will higher orders influence the performance of the grating.

If the real interfaces of material-air are taken into account during the calculation of the AS maps (in RCWA), the profile similar to that in Figure 6(b) is obtained. In this figure, an interference pattern caused by mentioned interfaces is present. It can be seen that the inclusion of the interfaces does not alter the overall profile of the AS map.

Until now mainly transmission gratings were discussed, now some important differences which apply for reflection gratings will be described.

As a consequence of a shorter spatial period in the case of the reflection gratings (Figure 7), the Bragg curves are inclined more to the angular axes. Moreover, because of the 90 deg rotational shift of the modulation planes, the Bragg curves are shifted of $90 \mathrm{deg}$ as well (on the angular axes). Both of these properties, consequently, lead to a continuous pattern at the apex of these curves and to a different selectivity with respect to the detuning via an angle or wavelength. If the angle is changed, the cross-section of the AS map is more along the profile of the Bragg condition and the selectivity has a broader peak than in the case of the wavelength change where the cross-section is oriented more perpendicular and has a narrower peak (Figure 7). The position in the AS map along the wavelength axis is influenced by a spatial period of the modulation planes.

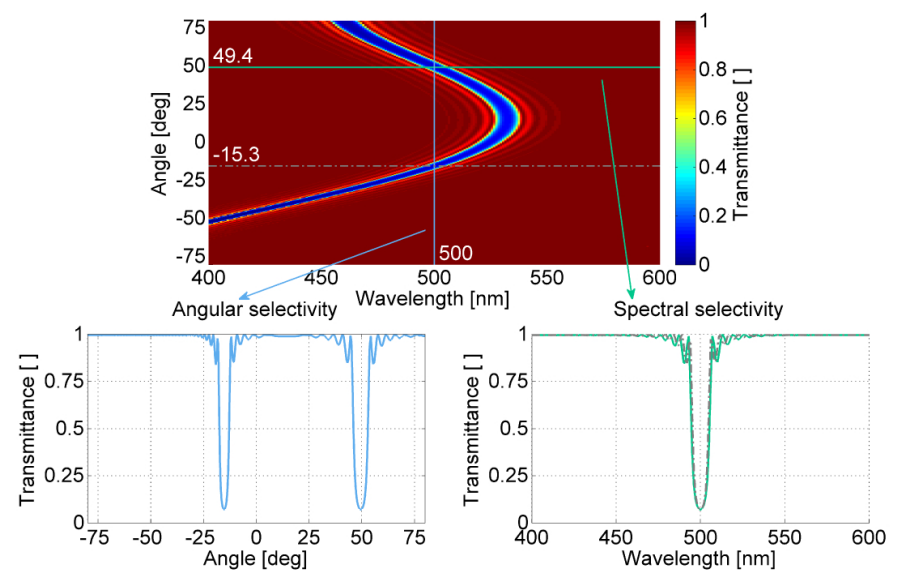

FIG. 7 AS map for a slanted reflection grating with indicated cross-sections - angular and spectral selectivity. Grating parameters: $\Lambda=175 \mathrm{~nm}, n_{0}=1.52, n_{1}=0.02$, $d=15 \mu \mathrm{m}, \psi=10 \mathrm{deg}$.

As in the case of the transmission gratings, where an angular window was apparent, here a spectral window can be extracted, similarly. This window is rather narrow and its limits are given by the perpendicular incidence (with respect to the planes of the modulation) on one side and by the angle of total reflection [28] on the other one if the in/out-coupling into the grating is not considered. For slanted in/out-coupling gratings, this spectral window broadens into shorter wavelengths but the angular windows starts to be limited.

For the slanted reflection gratings, the transmittance profile is again moving to the side of the map (with respect to the angle) where the shift is corresponding to the change of the slant angle. The profile gets broader for the side which moved into higher angles and narrower for the other one, as in the case of the transmission gratings. On top of that for higher slant angles, the grating can be used as a in/out-coupling. In the case of reflection gratings, this in/out-coupling (in Figure 7 for wavelength region $400-450 \mathrm{~nm}$ ) is possible for shorter wavelengths than is the wavelength corresponding to the angle of the total reflection (under the Bragg condition).

Aside from above mentioned overall effects, various grating properties and parameters can be extracted. From the AS map of the grating, the position and the angular-spectral progress of the Bragg condition (corresponds to minimums in the AS map) can be obtained for the first (especially) and higher diffraction orders. The curve along the Bragg condition in the angular-spectra plane indicates the spatial period of a grating and comparison among such curves can be used for a distinction of gratings with different periods. Orientation of the refractive index modulation planes, i.e. slant angle, is given by the shift of the position of the Bragg condition in the AS map with respect to the zero reconstruction angle (perpendicular incidence). The diffraction efficiency can be extracted from the AS map and, if only the first diffraction order is assumed (no higher orders are present or are not very pronounced), then, with the help of the theory, estimated values of the refractive index modulation and thickness of the grating can be calculated. For transmission gratings, it is possible to estimate the angular-spectral region where the grating pronounces the optimal reconstruction, is undermodulated or overmodulated. 


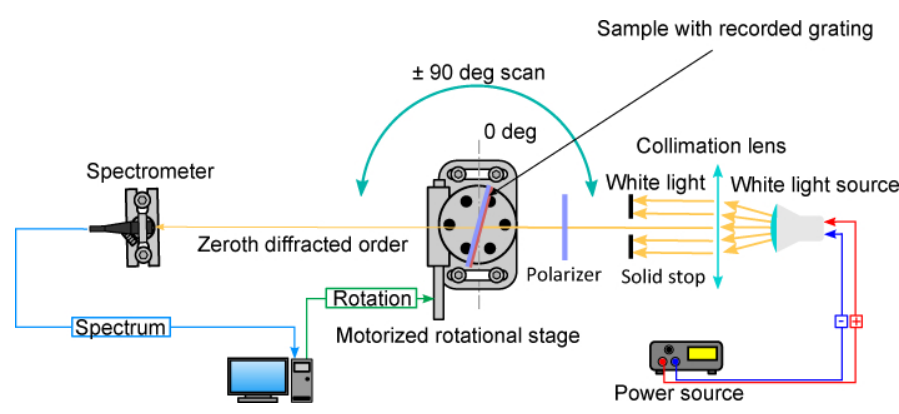

FIG. 8 Schematic illustration of a measurement setup for measuring of the AS maps.

Moreover, to a some degree, shrinkage, i.e. volume changes, can be assessed from the difference between the expected and obtained reconstruction parameters.

\section{MEASUREMENT SETUP}

For measurements of diffraction grating samples and for obtaining the AS maps, a measurement setup depicted in Figure 8 was used. The analyzed sample was positioned on a motorized rotational stage (Thorlabs CR1/M-Z7E) which was controlled via a computer. A collimated white light source (OceanOptics HL-2000) was used as a probe beam and the optimal diameter of this probe beam was selected by introducing a circular solid stop. The limitations of the size of the probe beam diameter are related to the dimensions and the shape of a sample grating. For a selection of a polarization of the probe beam (TE or TM), a linear polarizer was used. The response of a grating to this probe beam was analyzed by a standard fiber spectrometer (OceanOptics HR4000) in the zeroth (transmitted) order. The coupling into this fiber spectrometer was done by a focusing lens (i.e. a collimation fiber adapter). It is desirable to place the solid stop and the coupling lens as near as possible to the sample; nevertheless, the spatial separation of diffraction orders must be larger than the input aperture of the coupling lens.

For the measurement itself, a sample was positioned on the rotational platform under a 90 deg angle with respect to the probe beam (the angle between the normal to the surface and the probe beam). The planes of the refractive index modulation of a grating were oriented perpendicularly to the plane of incidence. Then the rotational stage performed a step wise rotation of $180 \mathrm{deg}$ with a chosen step size, e.g. $0.25 \mathrm{deg}$. For each step, the transmitted spectrum was collected and analyzed.

After the full rotation, the AS map can be constructed from the measured spectra and the knowledge of the angular position where these spectra were taken. The measurement, acquisition, and processing of data were controlled via an in-house developed control function in the Matlab ${ }^{\circledR}$ environment.

The reason for measuring only the zeroth (transmitted) order and not directly, for example, the first diffracted order consist in the fact that the position of the diffracted order changes during the scan and also spectral dispersion occurs; therefore, its measurement is more experimentally complicated. Moreover, in the diffraction order spectrum even higher orders, than only the zeroth and first one can be present and so the measurement and analysis of the zeroth order can provide us with additional information about the higher orders influence.

An important note is that this setup can measure and provide information of diffraction properties of any type of volume phase diffraction grating, including transmission, reflection, or their slated variants with coupling effects.

Measurements of the AS maps for the sample gratings were carried out in following way. At first, a reference sample, which consisted of a glass substrate and homogeneously exposed (no grating) material, was positioned at the rotational platform and measured for the full angular scan (due to practical reasons only the angular span $\pm 80 \mathrm{deg}$ was used for the analyzes). This measurement provided us with a correction (reference) data for a residual absorption, scattering, reflection and other losses, present in the recording material. In the second step, a sample grating itself was put on the stage and measured with the same angular resolution as the reference measurement. Obtained data were then corrected with the reference scan (note, the scattering inside the material caused by the presence of the grating itself was not fully corrected).

\section{RESULTS OF MEASUREMENT}

The AS maps with experimental approach presented in Section 4 were measured and analyzed for a wide range of recorded grating samples which were recorded into several different materials (typically AgBr, photopolymers, etc.). Here, only selected representative sample gratings, which appropriately illustrate many of the above mentioned effects and properties, will be considered. Material into which the representative gratings were recorded was a photopolymer recording system Bayfol HX [10]. This material was chosen because we tested it in our laboratory for its applicability in a recording of wide range of diffraction gratings and elements. The characteristics of sample gratings are given in Table 2.

Obtained results (TE polarization) for the transmission gratings with various grating parameters are shown in Figures 9-11, for the reflection gratings in Figure 12.

For both grating types, there is a good correspondence between the measured and simulated AS maps, especially in the character of these maps and main trends. Moreover, above mentioned effects and properties (see Section 3) hold true. Nevertheless, some differences are present, e.g. additional gratings are present.

From the Figure 9 it can be seen that the grating is of transmission type due to the position and character of the Bragg condition. Moreover, because there is no apparent shift to the side with respect to the angular axis (the AS map is symmetrical), the grating is non-slanted. Further, this figure presents the change of the AS maps for two different exposure dosages but the same grating parameters. It can be seen that in this case the higher exposure dosage led to a shift of the main decrease in the transmittance (first order) into longer wavelengths, higher overmodulation for shorter wavelengths, and more 


\begin{tabular}{cccccccc}
\hline Sample & $\begin{array}{c}\text { Grating } \\
\text { type }^{+}\end{array}$ & $\begin{array}{c}\mathrm{I} \\
{\left[\mathrm{mW} / \mathrm{cm}^{2}\right]}\end{array}$ & $\begin{array}{c}\mathrm{t} \\
{[\mathrm{s}]}\end{array}$ & $\begin{array}{c}\mathrm{E} \\
{\left[\mathrm{mJ} / \mathrm{cm}^{2}\right]}\end{array}$ & $\begin{array}{c}\lambda_{\text {rec }} \\
{[\mathrm{nm}]}\end{array}$ & $\begin{array}{c}\Lambda \\
{[\mathrm{nm}]}\end{array}$ & $\begin{array}{c}\psi \\
{[\mathrm{deg}]}\end{array}$ \\
\hline $\mathrm{A}$ & $\mathrm{T}$ & 0.9 & 8 & 7.2 & 532 & 700 & 90 \\
$\mathrm{~B}$ & $\mathrm{~T}$ & 9 & 8 & 72 & 532 & 700 & 90 \\
$\mathrm{C}$ & $\mathrm{T}$ & 9 & 8 & 72 & 532 & 600 & 85 \\
$\mathrm{D}$ & $\mathrm{T}$ & 9 & 8 & 72 & 532 & 700 & 80 \\
$\mathrm{E}$ & $\mathrm{T}$ & 9 & 8 & 72 & 532 & 900 & 90 \\
$\mathrm{~F}$ & $\mathrm{R}$ & 20 & 8 & 160 & 532 & 175 & 0 \\
$\mathrm{G}$ & $\mathrm{R}$ & 2 & 8 & 16 & 633 & 208 & 15 \\
$\mathrm{H}$ & $\mathrm{R}$ & 2 & 8 & 16 & 633 & 208 & 0 \\
\hline
\end{tabular}

$\mathrm{t}-\mathrm{T}$ and $\mathrm{R}$ represent transmission and reflection gratings respectively.

TABLE 1 Measured and analyzed samples with their intended recording parameters (overall intensity I, exposure time t, exposure dosage $E$, recording wavelength $\lambda_{\text {rec }}$, spatial period $\Lambda$, and slant angle $\psi$ ).

a)

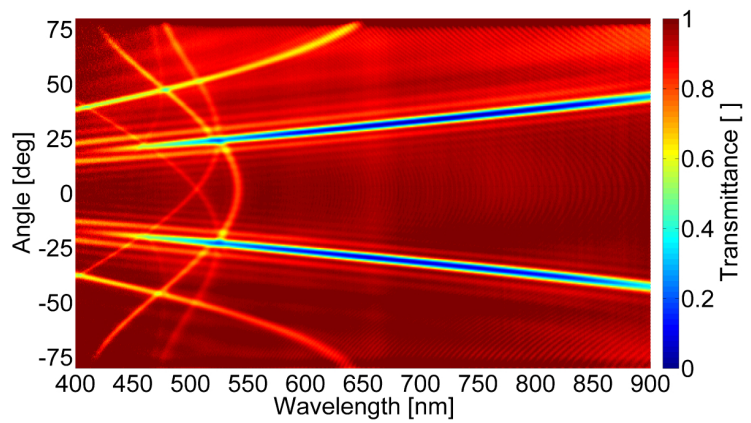

b)

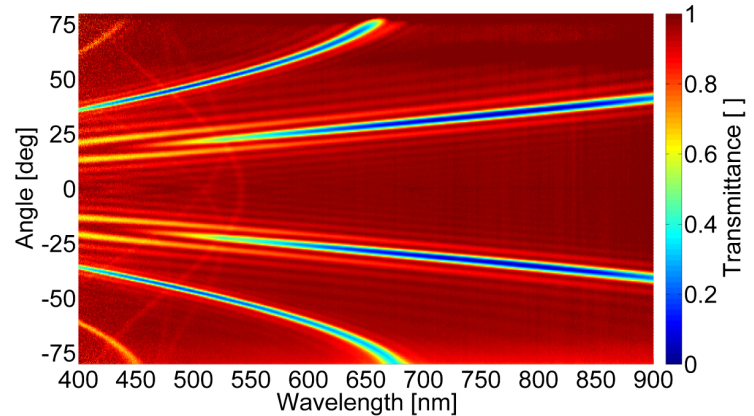

FIG. 9 Measurement of non-slanted transmission gratings. Intended recording parameters are shown in Table 2.

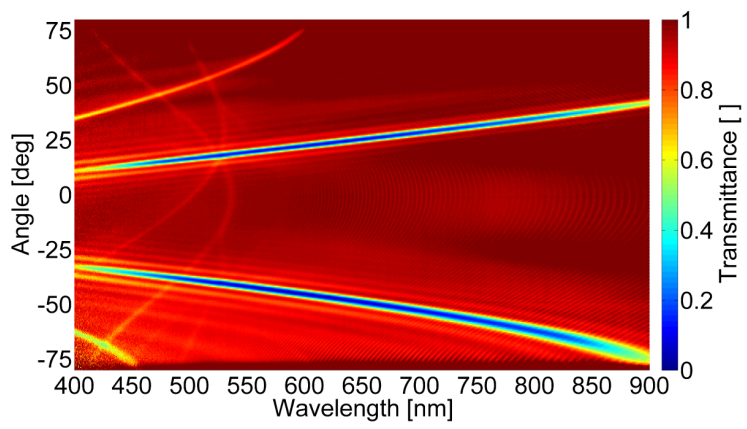

FIG. 10 Measurement of slanted transmission grating (Sample C). Intended recording parameters are given in Table 2.

pronounced higher diffraction orders (second and third). Figure 10 illustrates an effect of the change of the slant angle and the corresponding shift and bending of the profile to the side with respect to the angle axis. Figure 11 presents obtained angular and spectral cross-sections positioned at optimal reconstruction.
In the AS maps, higher (second and third) diffraction orders are clearly visible. Based on their analysis, it is possible to extract information about higher modulation orders. The theory, where the higher modulation orders were not considered, did not predicted such strong efficiencies of the higher diffraction orders. Consequently, it can be concluded that the main cause for this behavior is presence of the higher modulation orders of the refractive index (in an agreement with literature [21]). Moreover, the character and behavior of these diffraction orders for a wide range of reconstruction angles and wavelengths is apparent.

It is possible to extract optimal reconstruction parameters (indicated in Figure 11) and assess behavior for different parameters from the measured maps. The effect of overmodulation for some reconstruction parameters is apparent as well.

Presented figures with the AS maps representing transmission gratings can be used for indication and assessment of presence of parasite gratings (e.g. reflection) which were formed as a consequence of an unintended reflections (e.g. back reflection) and interferences of recording beams. In presented figures (e.g. wavelength region 400 - $550 \mathrm{~nm}$ in Figure 9(a)), there are at least three such parasite gratings visible. The first, which is symmetrically centered, is caused by an interference of the incident reference wave and its back reflection (the same applies for a signal wave). The second and third parasite gratings, which are positioned at the first transmission diffraction order, originated through an interference between the incident signal/reference wave and the back reflected reference/signal wave. In some figures (e.g. Figure 9(a)), additional parasite gratings positioned at the higher transmission diffraction orders are present as well. In single angular or wavelength scans these parasite gratings would be difficult to observe and distinguish.

It is apparent from the continuous pattern of the Bragg condition in Figure 12 that in these cases reflection gratings were measured. For these reflection gratings, the shape of the maps is in agreement with the theory, but the values of the transmittance itself can deviate to some small extend. Theory indicates (for TE polarization) that for shorter wavelengths the grating should be more efficient, but in the measurements, this character deviates from this expected behav- 


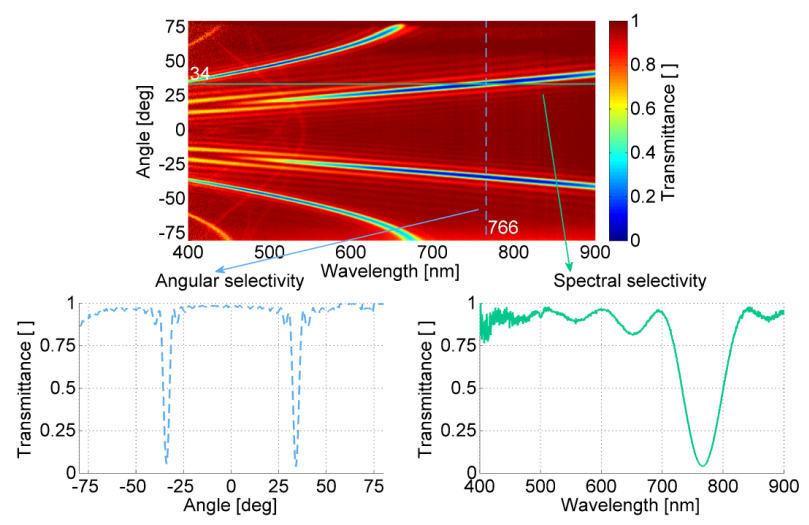

FIG. 11 Measurement of transmission grating (sample B) with indicated angular and spectral selectivities positioned at optimal reconstruction. Intended recording parameters are shown in Table 2.

a)

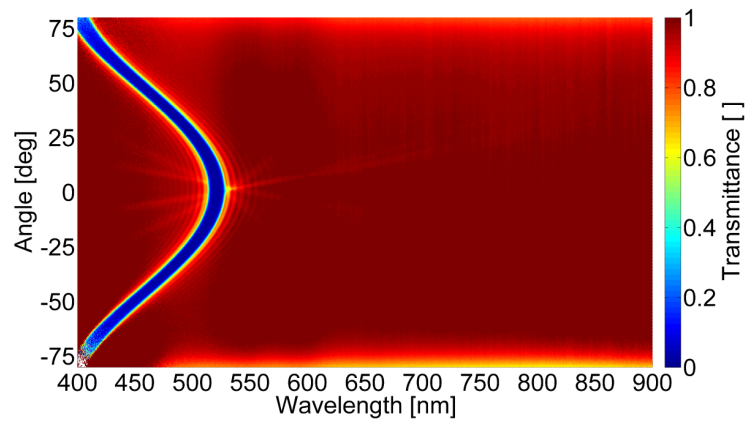

b)

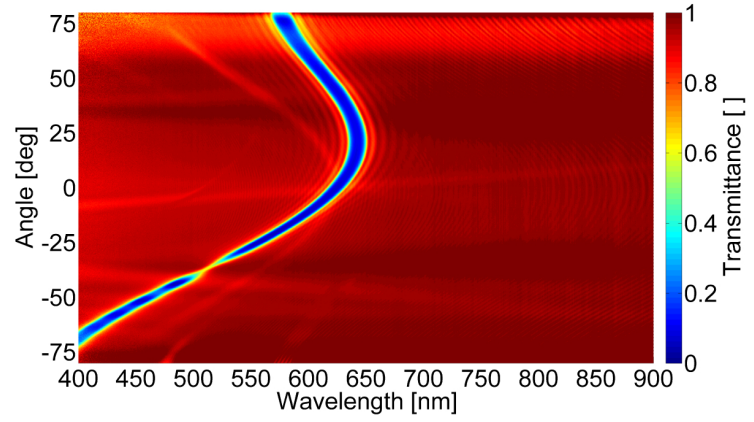

FIG. 12 Measurements of a) non-slanted and b) slanted reflection gratings. Intended recording parameters are in Table 2.

ior for angles larger than $\pm 50 \mathrm{deg}$ in Figure 12(a). This discrepancy can be caused by large measured angles and by the light source used in the measurements which has lower intensity in the region below $450 \mathrm{~nm}$, and so the useful data can be compromised by a noise. Moreover, such behavior can be a consequence of a polarization mix as well. The increase in the transmittance in the case of the grating in Figure 12(b) around the wavelength $510 \mathrm{~nm}$ and angle $-37.5 \mathrm{deg}$ is caused by a diffraction of the in-coupled wave in the direction of the original beam. Apart form this behavior, the sample grating $\mathrm{G}$ has exhibited a good correspondence to the theory.

Table 1 presents obtained grating parameters (central wavelength $\lambda_{\text {opt }}$ and angle $\Theta_{\text {opt }}$ of optimal reconstruction, slant angle $\psi$, spatial period $\Lambda$, refractive index modulations $n_{1}, n_{2}$, and angular and spectral windows) of the sample diffraction gratings (transmission, reflection). The approach to the extraction was described in Section 3. For the transmission grat- ings, the optimal reconstruction parameters and corresponding grating parameters were obtained; for reflection gratings these parameters were extracted for a one chosen wavelength. It is good to note that the comparison between the obtained and intended parameters (Table 2) shows some differences in the case of the spatial period and slant angle which is consequence of the recording adjustment (e.g. the error in the spatial period can be relatively large due to a small change of the recording angle) and possible shrinkage of the recording material.

\section{CONCLUSIONS}

In this paper, an implementation of the measurement method, based on AS map scans, was presented and discussed; moreover, this method was tested and its results were compared with the theory. A striking agreement between theoretical predictions and the measurement results was observed. Based on this agreement using this complex evaluation method, many important properties of diffraction gratings are possible to extract. This clearly helps in many practical applications.

With the help of the AS map, it is possible to quickly distinguish between transmission and reflection gratings (or their combination) and to obtain the spatial period, angle of modulation planes, optimal reconstruction parameters, etc. For example, the slant angle is given by a non-symmetry of phase synchronizing condition (the Bragg condition) in the AS map, the spatial period is easily determined by the angular character of the AS map, etc.

It turns out that, for longer wavelengths, it is not possible for some gratings to fulfill the Bragg condition which is again readily seen from the AS map (the spectral window). This property is pronounced for the reflection gratings in the visible spectral range and is dependent on the spatial period of the grating. Moreover, from the spectral or angular crosssections, it is possible to obtain selectivity curves for a given angle or wavelength.

This method clearly find an application for a characterization and control of produced diffraction volume gratings recorded into photopolymer systems but can be used for other recording materials as well.

\section{ACKNOWLEDGEMENTS}

This work was supported by the Ministry of Education, Youth and Sports of the Czech Republic under Project NPU I LO1206, and by the Grant Agency of the Czech Technical University in Prague, grant No. SGS13/221/OHK4/3T/14. Furthermore, we would like to thank Bayer MaterialScience AG for providing of samples of the Bayfol HX photopolymer recording material, and our colleague Pavel Kwiecien for providing the computer implementation of the Rigorous Coupled Wave Analysis procedure. 


\begin{tabular}{lllllllllll}
\hline Sample & $\begin{array}{l}\text { Grating } \\
\text { type }^{\dagger}\end{array}$ & $\begin{array}{l}\lambda_{\text {opt }} \\
{[\mathrm{nm}]}\end{array}$ & $\begin{array}{l}\Theta_{\text {opt }}(\mathrm{out}) \\
{[\mathrm{deg}]}\end{array}$ & $\begin{array}{l}\psi \\
{[\mathrm{deg}]}\end{array}$ & $\begin{array}{l}\Lambda \\
{[\mathrm{nm}]}\end{array}$ & $\begin{array}{l}\mathrm{d} \\
{[\mu \mathrm{m}]}\end{array}$ & $\begin{array}{l}n_{1} \\
\left(@ \lambda_{\text {opt }}\right)\end{array}$ & $\begin{array}{l}n_{2} \\
(@ 532 \mathrm{~nm})\end{array}$ & $\begin{array}{l}\mathrm{AW}^{* o} \\
{[\mathrm{deg}]}\end{array}$ & $\begin{array}{l}\mathrm{SW}^{* *} \\
{[\mathrm{~nm}]}\end{array}$ \\
\hline $\mathrm{A}$ & $\mathrm{T}$ & 730 & $33.5 /-34.5$ & 89.7 & 653 & 15 & 0.019 & 0.003 & $18-43.5$ & $\mathrm{FR}$ \\
$\mathrm{B}$ & $\mathrm{T}$ & 766 & \pm 34 & 90 & 685 & 15 & 0.021 & 0.008 & $17.5-41.5$ & $\mathrm{FR}$ \\
$\mathrm{C}$ & $\mathrm{T}$ & 592 & $21.5 /-41$ & 83.1 & 547 & 15 & 0.015 & 0.003 & $11-42$ & $\mathrm{FR}$ \\
$\mathrm{D}$ & $\mathrm{T}$ & 696 & $17 /-53$ & 79.7 & 628 & 15 & 0.019 & 0.006 & $3-28.5$ & FR \\
$\mathrm{E}$ & $\mathrm{T}$ & 639 & $20.5 /-21$ & 89.8 & 902 & 15 & 0.019 & 0.011 & $13-30$ & FR \\
$\mathrm{F}$ & $\mathrm{R}$ & $500^{\ddagger}$ & $25.5 /-24.5$ & 0.3 & 171 & 12.5 & 0.033 & & \pm 90 & $404-520$ \\
$\mathrm{G}$ & $\mathrm{R}$ & $600^{\ddagger}$ & $58.8 /-11$ & 13.5 & 211 & 13 & 0.026 & & $-23-90$ & $570-642$ \\
$\mathrm{H}$ & $\mathrm{R}$ & $600^{\ddagger}$ & $26.5 /-22.5$ & 1.24 & 205 & 13 & 0.025 & & \pm 0 & $470-625$ \\
\hline
\end{tabular}

†- $\mathrm{T}$ and $\mathrm{R}$ represent transmission and reflection gratings respectively; $\ddagger$ - for reflection gratings these values are not optimal, but values for which the parameters were evaluated; ${ }^{*}$ and ${ }^{* *}$ - angular (AW) and spectral (SW) window respectively, for which the first diffraction order can be reconstructed and propagated in air (Bragg condition); FR stands for a "full range" (400-900 nm for transmision gratings); o - AW for T gratings only for one Bragg condition.

TABLE 2 Extracted parameters of the studied gratings.

\section{References}

[1] G. Odian, Principles of Polymerization (4th Edition, WileyInterscience, Haboken, 2004).

[2] J. T. Sheridan, M. Downey, and F. T. O’Neill, “Diffusion-based model of holographic grating formation in photopolymers: generalized non-local material responses," J. Opt. A-Pure Appl. Optics 3, 477-488 (2001).

[3] M. R. Gleeson, and J. T. Sheridan, "A review of the modelling of free-radical photopolymerization in the formation of holographic gratings," J. Opt. A-Pure Appl. 0p. 11, 024008 (2009).

[4] H. Li, Y. Qi, and J. T. Sheridan, “Three-dimensional extended nonlocal photopolymerization driven diffusion model. Part II. Photopolymerization and model development," J. Opt. Soc. Am. B 31, 2648-2656 (2014).

[5] S. Gallego, C. Neipp, M. Ortuño, A. Beléndez, E. Fernández, and I. Pascual, "Analysis of monomer diffusion in depth in photopolymer materials," Opt. Commun. 274, 43-49 (2007).

[6] M. Moothanchery, V. Bavigadda, V. Toal, and I. Naydenova, "Shrinkage during holographic recording in photopolymer films determined by holographic interferometry," Appl. Optics 52, 8519-8527 (2013).

[7] I. Naydenova, R. Jallapuram, R. Howard, S. Martin, and V. Toal, "Investigation of the diffusion processes in a self-processing acrylamide-based photopolymer system," Appl. Optics 43, 2900-2905 (2004).

[8] F.-K. Bruder, F. Deuber, T. Facke, R. Hagen, D. Honell, D. Jurbergs, M. Kogure, et al., "Full-Color Self-processing Holographic Photopolymers with High Sensitivity in Red-The First Class of Instant Holographic Photopolymers," J. Photopolym. Sci. Tec. 22, 257-260 (2009).

[9] T. Smirnova, L. Kokhtich, O. Sakhno, and J. Stumpe, “Holographic nanocomposites for recording polymer-nanoparticle periodic structures: I. General approach to choice of components of nanocomposites and their holographic properties," Opt. Spectrosc. 110, 129-136 (2011).

[10] H. Berneth, F.-K. Bruder, T. FÃd'cke, D. Jurbergs, R. Hagen, D. Hönel, T. Rölle, and G. Walze, "Bayfol HX photopolymer for full-color transmission volume Bragg gratings," Proc. SPIE 9006, 900602 (2014).

[11] M. R. Gleeson, S. Liu, and J. T. Sheridan, "Improvement of photopolymer materials for holographic data storage," J. Mater. Sci. 44, 6090-6099 (2009).
[12] N. Suzuki, and Y. Tomita, "Real-time phase-shift measurement during formation of a volume holographic grating in nanoparticledispersed photopolymers," Appl. Phys. Lett. 88, 011105 (2006).

[13] S. Callego, A. Marquez, F. J. Guardiola, M. Riquelme, R. Fernandez, I. Pascual, and A. Belendez, "Linearity in the response of photopolymers as optical recording media," Opt. Express 21, 10995-11008 (2013).

[14] F. T. O'Neill, J. R. Lawrence, and J. T. Sheridan, “Thickness variation of self-processing acrylamide-based photopolymer and reflection holography," Opt. Eng. 40, 533-539 (2001).

[15] M. Ortuno, M. Riquelme, S. Gallego, A. Márquez, I. Pascual, and A. Beléndez, "Overmodulation Control in the Optimization of a $\mathrm{H}$ PDLC Device with Ethyl Eosin as Dye," Int. J. Polym. Sci. 2013, 8 (2013).

[16] M. Květoň, A. Havránek, P. Fiala, and I. Richter, “Polymer holography I - Method and experiment," Ploym. Bull. 58, 253-259 (2007).

[17] S. Gallego, M. Ortuno, C. Neipp, C. Garcia, A. Belendez, and I. Pascual, "Overmodulation effects in volume holograms recorded on photopolymers," Opt. Commun. 215, 263-269 (2003).

[18] M. Moothanchery, I. Naydenova, and V. Toal, "Study of the shrinkage caused by holographic grating formation in acrylamide based photopolymer film," Opt. Express 19, 13395-13404 (2011).

[19] P. Trochtchanovitch, N. Kostrov, E. Goulanian, A. F. Zerrouk, E. Pen, and V. Shelkovnikov, "Method of characterization of effective shrinkage in reflection holograms," Opt. Eng. 43, 1160-1168 (2004).

[20] H. Kogelnik, "Coupled Wave Theory for Thick Hologram Gratings," Bell Syst. Tech. J. 48, 2909-2947 (1969).

[21] R. Alferness, "Analysis of propagation at the second-order Bragg angle of a thick holographic grating," J. Opt. Soc. Am. 66, 353-362 (1976).

[22] G. Zhao, and P. Mouroulis, "Second order grating formation in dry holographic photopolymers," Opt. Commun. 115, 528-532 (1995).

[23] T. Gaylord, and M. Moharam, "Analysis and applications of optical diffraction by gratings," Proc. IEEE 73, 894-937 (1985).

[24] M. G. Moharam, D. A. Pommet, E. B. Grann, and T. K. Gaylord, "Stable implementation of the rigorous coupled-wave analysis for surface-relief gratings: enhanced transmittance matrix approach," J. Opt. Soc. Am. A 12, 1077-1086 (1995).

[25] I. Richter, Z. Ryzí, and P. Fiala, "Analysis of binary diffraction gratings: Comparison of different approaches," J. Mod. Optic. 45, 1335-1355 (1998). 
[26] M. G. Moharam, E. B. Grann, D. A. Pommet, and T. K. Gaylord, "Formulation for stable and efficient implementation of the rigorous coupled-wave analysis of binary gratings," J. Opt. Soc. Am. A 12, 1068-1076 (1995).

[27] P. Vojtís̃ek, and M. Květoň, "Monitoring of overmodulation effect in high efficient transmission gratings produced in photopolymers," Proc. SPIE 9450, 945011-945018 (2015).
[28] P. Vojtís̃ek and M. Květoň, "Real-time direct measurement of diffraction efficiency of reflection gratings in photopolymer recording materials," Proc. SPIE 9442, 944211-944218 (2015). 